\title{
A Study on the Optimal Receiver Impedance for SNR Maximization in Broadband PLC
}

\author{
Massimo Antoniali, Andrea M. Tonello, and Fabio Versolatto \\ Dipartimento di Ingegneria Elettrica Gestionale e Meccanica (DIEGM), Università di Udine, 33100 Udine, Italy \\ Correspondence should be addressed to Andrea M. Tonello; tonello@uniud.it
}

Received 27 July 2012; Revised 31 October 2012; Accepted 13 December 2012

Academic Editor: Moises V. Ribeiro

Copyright (C) 2013 Massimo Antoniali et al. This is an open access article distributed under the Creative Commons Attribution License, which permits unrestricted use, distribution, and reproduction in any medium, provided the original work is properly cited.

\begin{abstract}
We consider the design of the front-end receiver for broadband power line communications. We focus on the design of the input impedance that maximizes the signal-to-noise ratio (SNR) at the receiver. We show that the amplitude, rather than the power, of the received signal is important for communication purposes. Furthermore, we show that the receiver impedance impacts the amplitude of the noise term. We focus on the background noise, and we propose a novel description of the noise experienced at the receiver port of a PLC network. We model the noise as the sum of four uncorrelated contributions, that is, the active, resistive, receiver, and coupled noise components. We study the optimal impedance design problem for real in-home grids that we assessed with experimental measurements. We describe the results of the measurement campaign, and we report the statistics of the optimal impedance. Hence, we study the best attainable performance when the optimal receiver impedance is deployed. We focus on the SNR and the maximum achievable rate, and we show that power matching is suboptimal with respect to the proposed impedance design approach.
\end{abstract}

\section{Introduction}

The communication technology that exploits the power delivery network to convey data is commonly referred to as power line communication (PLC). PLC is broadly deployed and, recently, it has been recognized as a key technology to enable the communication within the smart grid. The last node of the smart grid is the home, where PLC is suitable for both home entertainment, with datarates of about $200 \mathrm{Mbps}$, and homeautomation, with lower data-rates but higher robustness and reliability.

The design of the PLC transceiver is a challenging task due to the severe attenuation, fading effects, and noise impairments that characterize the communication media. In other application scenarios, as wireless, the transceivers are designed to fulfill the maximum power transfer condition. Basically, the maximum power transfer is achieved under complex matching conditions, that is, when the internal impedance of the transmitter and the input impedance of the receiver are the complex conjugate of the characteristic impedance of the transmission medium. In wireless, this corresponds to the input impedance of the antenna. Indeed, the absence of reflections and stationary waves is obtained under simple (not complex) matching conditions, that is, when the impedance of the transmitter and the receiver are equal to the characteristic impedance of the transmission medium. Reflections and stationary waves are not desired. The reflected waves yield to multiple delayed echoes in the channel impulse response and they can be either due to the multipath nature of the channel or due to the unmatched termination nodes. Matching ensures the absence of the latter type of reflections. The stationary waves yield to voltage values along the line that are higher than necessary and that may damage the transmission medium, especially in wireline communications as, for instance, over coaxial cables.

When the characteristic impedance of the medium is real, the maximum power transfer and the absence of reflections are ensured by the same matching conditions. In the following, we simply indicate with impedance matching the receiver design that enables the maximum power transfer. In wireless, impedance matching can be easily satisfied by letting the characteristic impedance of the antenna and the 
cables, the internal impedance of the transmitter and the input impedance of the receiver be equal to the reference value of $50 \Omega$.

In PLC, impedance matching is typically achieved through the use of impedance matching networks, so that the received signal power is maximized. Basically, the matching network is a loss-less network of lumped elements whose input impedance is matched to that of the power delivery network. The latter is frequency dependent and it varies significantly from outlet to outlet. Furthermore, it may exhibit a time-varying behavior [1]. Several matching networks were presented in the literature for PLC, and their use was demonstrated to be beneficial in terms of an increase of the received signal power $[2$, Ch. 4] $[3,4]$. However, from a data transmission point of view, it is important to maximize the signal-to-noise ratio (SNR) and not only the signal power.

The effective formulation of the SNR is in signal amplitude rather than in power terms. The reason is that the analog front-end of the PLC receiver is designed to convert the analog amplitude of the received signal into a digital sample stream. In this respect, the amplitude of the received signal is more important than its power, though the two quantities are related once the receiver impedance is given.

In PLC, the maximum power transfer condition does not imply the maximization of the SNR in amplitude terms because power matching may turn into a higher noise contribution. Concerning an in-home network, the noise injected by the household devices is attenuated by the insertion loss of the path followed to reach the receiver outlet. In general, the latter is different from that followed by the communication signal. The receiver impedance affects the insertion loss, and common impedance matching techniques do not take into account the impact of the receiver impedance on the insertion loss experienced by the noise. It follows that impedance matching may reduce the attenuation of the noise path, thus increasing the noise amplitude at the receiver port. Furthermore, the receiver impedance itself contributes to the increase of the amplitude of the noise.

In this work, we discuss the optimal design of the receiver impedance that enables achieving the maximum SNR in broadband PLC. We formulate the SNR in terms of amplitude and we focus on in-home networks.

Firstly, we propose an analytic description of the noise as the sum of multiple contributions. We consider the resistive noise of the network by itself, the noise injected by the household devices, and the noise introduced by the receiver impedance. From the experimental evidence, we show that the noise injected by the household devices, namely, the active noise, dominates among all noise contributions.

Then, we formulate the SNR as a function of the receiver impedance. To this aim, we assume the transmitter impedance to be constant and known. We study the convexity of the optimization problem and we derive the optimal receiver impedance that maximizes the SNR at each single frequency.

Finally, we validate the results for real-life in-home networks. We exploit the results of an experimental measurement campaign that we carried out in Italy, where we collected more than 1200 channel responses in different premises. For each site, we performed measurements between all couples of available outlets in the $1.5-100 \mathrm{MHz}$ frequency range. The measured database is useful to provide a realistic description of the frequency response and the line impedance that characterizes the signal paths.

In this work, we focus on the time invariant description of the network because, from the experimental evidence, we observed that very little (or inexistent) time variation was present in the sites that we considered, namely, in the order of few dBs. More in general, the PLC channel can be periodically time variant. In such a case, an extension of our analysis can be obtained under the assumption that both the channel and the noise exhibit a slow periodic variation that is synchronous with the mains. Thus, the mains period can be divided into short time intervals (slots) during which we can reasonably assume both the channel and the noise to be time invariant. Hence, we can apply the analysis that we propose in this work to each single slot.

We study the attainable SNR improvement when the optimal receiver impedance is used. We compare the results to the case of impedance matching, when the receiver impedance is constant and equal to the reference value of $50 \Omega$, and when it is constant and equal to $1 \mathrm{k} \Omega$. The latter case is representative of a receiver with high input impedance.

The remainder of the work is as follows. In Section 2, we describe the model of the power delivery network. In Section 3, we overview the transceiver design, with emphasis on the receiver side. Then, in Section 4, we formulate the optimization problem, and, in Section 5, we study its convexity. In Section 6, we present several numerical results. Basically, we study the optimal receiver impedance for a real scenario. Furthermore, we provide some details on the measurements. Then, we study the performance in terms of SNR and achievable rate and we compare the results to the ones that we would obtain for the other receiver impedance designs. Finally, the conclusions follow.

\section{Power Delivery Network Model}

PLC experiences high attenuation and deep fading effects that are a function of the loads and the layout of the network. We focus on in-home networks. In [5], we presented an accurate model of the in-home power delivery network. Basically, inhome networks exhibit a treelike structure and the outlets are the termination nodes. The outlets are fed by the power line cables which resemble the branches of the network. Between any pair of outlets, only one electrical path is possible.

From a data transmission perspective, we can abstract the power delivery network to obtain an equivalent representation that is suitable for the SNR analysis of the following sections. In Figure 1, we show the model. We represent the power delivery network as an $\mathcal{O}$-port network, where $\mathcal{O}$ is the number of outlets. The transmitter and the receiver are connected to two ports of the network, namely, ports $t$ and $r$. We model the transmitter as a real voltage source with its own internal impedance, and the receiver as a passive load. The household appliances are connected to the remaining ports of the network. They inject noise, and we model them as 


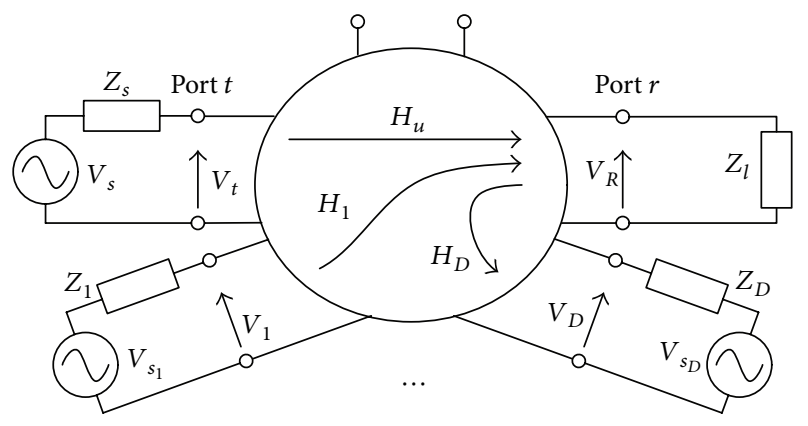

FIGURE 1: Equivalent model of the power delivery network.

noise sources, namely, real voltage generators with their own internal impedance. In general, the number of household appliances is $D \leq \mathcal{O}$ because some outlets may be not in use, as shown in the uppermost part of Figure 1.

The signal path between two ports of the network is characterized by the channel frequency response (CFR). We refer to the CFR as the ratio, in the frequency domain, between the voltage at the output and input ports.

We introduce the following notation. We denote the complex amplitude of the source signal, the internal impedance of the transmitter, the complex amplitude of the voltage at the transmitter port, the complex amplitude of the voltage at the receiver port, and the input impedance of the receiver with $V_{s}(f), Z_{s}(f), V_{t}(f), V_{R}(f)$, and $Z_{\ell}(f)$, respectively. All the quantities are defined in the frequency domain, at frequency $f$. Concerning the noise, we use the subscript $\{\cdot\}_{d}$ to denote the $d$ th source of noise, where $d=1, \ldots, D$, and we denote the complex amplitude of the $d$ th noise source and its internal impedance with $V_{s_{d}}(f)$ and $Z_{d}(f)$, respectively, and the voltage amplitude at the port where the $d$ th noise source is connected with $V_{d}(f)$. Furthermore, we note that all impedances are, in general, noisy and thus they generate a thermal noise contribution. Finally, we denote the CFR between the transmitter and the receiver port with $H_{u}(f)$, and between the port where the $d$ th noise source is connected and the receiver port with $H_{d}(f)$.

\section{PLC Transceiver Design}

We consider the analog front-end (AFE) of the transceiver. At the transmitter side, the AFE amplifies the signal. The final amplification stage is the line driver. In this work, we do not consider the impairments related to the design of the line driver and we model it as a real voltage generator with an internal impedance $Z_{s}$. To obtain the numerical results of Section 6, we simply neglect it; that is, we let $Z_{s}=0 \Omega$. The assumption is validated by the fact that the output impedance of the transmitter stage of commercially available broadband PLC transceivers is typically low, for example, $3 \Omega[6]$.

At the receiver side, the AFE processes the received signal to make it suitable for the analog-to-digital (AD) conversion. The final goal is to convert the (analog) amplitude of the received signal into a digital sample stream. Thus, the focus is

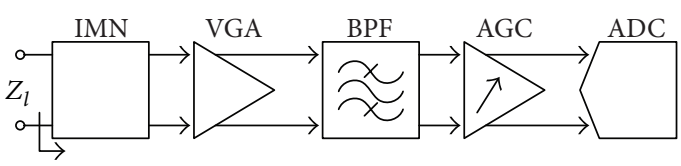

FIGURE 2: Block diagram of the PLC receiver AFE [6].

on the amplitude of the received signal, rather than its power, and this motivates the formulation of the SNR in amplitude terms. Furthermore, to preserve the signal amplitude, the input impedance of the analog-to-digital converter (ADC) circuit is typically high [7].

At the receiver, the AFE consists of several blocks. In Figure 2, we show the schematic diagram [6]. The variable gain amplifier (VGA) is followed by a band-pass filter (BPF) and an adaptive gain control (AGC). A low-noise amplifier (LNA) can be used to substitute the VGA, and an additional ad hoc amplifier can be deployed to drive the $\mathrm{AD}$ converter instead of the AGC [8]. The gain of the AGC is adjusted dynamically to ensure always the optimum signal level for $\mathrm{AD}$ conversion and the maximum dynamic range [6].

Practical receiver schemes adopt matching techniques to interface the blocks of the AFE. In wireless, where the communications are in the range of $\mathrm{GHz}$, the reference impedance value is $50 \Omega$ [9], and the advantages provided by impedance matching are the following. Firstly, since the reference impedance is real, matching enables both the maximum transfer of power and the absence of reflections at the receiver port. The latter condition is desired to avoid multipath fading exceeding that amenable closely to the channel. Secondly, matching simplifies the design of the LNA and the BPF. The noise figure (NF) of the LNA is a function of the impedance that is seen at the input and output port of the amplifier [10]. By letting the impedance be equal to a reference value, the LNA can be optimized to exhibit the lowest NF. Similarly, the behavior of the BPF is a function of the impedance seen by the filter at the input and output ports. The BPF is designed to operate in matching conditions, and its frequency behavior, that is, the pass-band and the stopbands, may vary significantly otherwise [9].

In PLC, the transmission interests the frequency range below $100 \mathrm{MHz}$, where the presence of reflected waves can be tolerated to preserve the amplitude of the received signal. Therefore, the interface between the blocks can be designed in high-impedance mode.

The use of an impedance matching network (IMN) before the AFE provides some benefits. Strictly, the matching network allows the receiver to be matched to the complex and frequency-dependent impedance of the power delivery network, regardless of the input impedance of the first stage of the AFE. In the literature, the design of the matching network was aimed at obtaining the maximum transfer of power from the network $[3,4]$. Alternative solutions are possible. In this respect, we propose the use of the matching network to obtain the optimal receiver impedance that lets the SNR be maximum, as we describe in the following sections. Basically, we model the IMN as an impedance $Z_{\ell}$ in parallel with the first stage of the AFE, and we assume the first stage to exhibit 
an infinite input impedance. Therefore, the resultant input impedance of the transceiver is equal to $Z_{\ell}$.

\section{SNR Formulation}

We follow the notation of Figure 1. We study the SNR at the receiver port $r$ when the transmitter is connected to port $t$, and $D$ noise sources are connected to the remaining ports of the network. At the receiver port, $V_{R}(f)$ is the sum of the signal of interest $V_{u}(f)$ and the noise $V_{n}(f)$, that is, $V_{R}(f)=$ $V_{u}(f)+V_{n}(f)$. We formulate the SNR in amplitude terms, as the power spectral density (PSD) of $V_{u}(f)$ divided by the PSD of $V_{n}(f)$. It reads

$$
\Gamma(f)=\frac{P_{V_{u} V_{u}}(f)}{P_{V_{n} V_{n}}(f)} .
$$

To obtain (1), we assume the signals to be stationary and continuous in time, and we note that the PSD of the generic signal $x(t)$, limited in power, can be computed as [11]

$$
P_{X X}(f)=\lim _{T \rightarrow \infty} \frac{\overline{\left(\left|X_{T}(f)\right|^{2}\right)}}{T}\left[\mathrm{~V}^{2} / \mathrm{Hz}\right],
$$

where $X_{T}(f)$ is the Fourier transform of $x(t)$ on a finite time interval of length $T$, and $\overline{(\cdot)}$ denotes the statistical expectation.

Now, we study the terms in (1). Firstly, let us consider the signal of interest. According to the notation of Figure 1, we explicit it as

$$
P_{V_{u} V_{u}}(f)=\left|H_{u}\left(Z_{\ell}, f\right)\right|^{2} P_{V_{t} V_{t}}(f)\left[\mathrm{V}^{2} / \mathrm{Hz}\right]
$$

Hence, let us focus on the noise term. In this work, we limit the study to the stationary noise components, and we explicit the PSD of the noise at the receiver port, namely, $P_{V_{n} V_{n}}(f)$ as the sum of contributions due to four noise terms. They are the active noise $V_{a}(f)$, the resistive noise $V_{r}(f)$, the receiver noise $V_{\ell}(f)$, and the coupled noise $V_{c}(f)$. The latter component models the radio disturbances that are captured by the wiring through coupling. We assume the coupled noise to be independent from the receiver impedance. Thus, we can neglect the impact of the coupled noise on the design of the optimal receiver impedance, and we let $P_{V_{c} V_{c}}(f)=0$. Furthermore, we assume the noise terms to be uncorrelated with zero mean. It follows

$$
P_{V_{n} V_{n}}(f)=P_{V_{a} V_{a}}(f)+P_{V_{r} V_{r}}(f)+P_{V_{e} V_{e}}(f)\left[\mathrm{V}^{2} / \mathrm{Hz}\right] .
$$

In the next sections, we describe the noise terms in (4) and we propose an analytical expression to model them, we introduce and motivate the approximations that allow us to formulate and solve the SNR maximization problem and, finally, we present the final SNR expression.

4.1. Active Noise. The active noise is generated by the powersupply circuitry of the household appliances that are connected to the power delivery network. We model the household appliances as real and independent voltage generators.

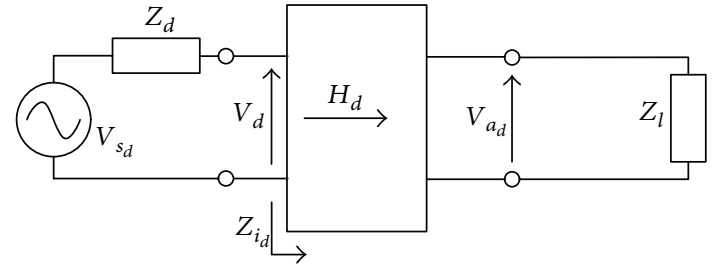

(a)

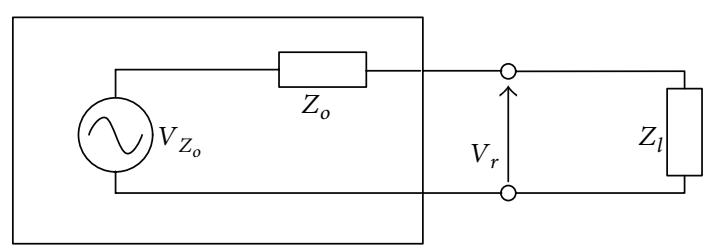

(b)

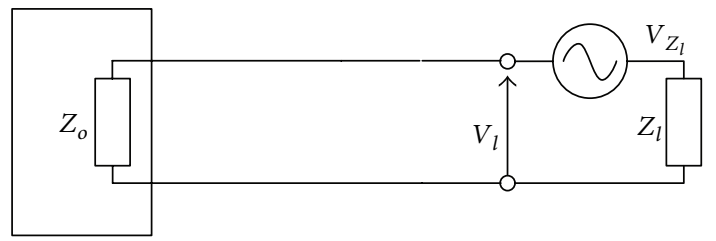

(c)

FIgURE 3: Equivalent models for the active, resistive, and receiver noise terms.

We show the equivalent model in Figure 3(a). The active noise at the receiver port reads

$$
\begin{aligned}
P_{V_{a} V_{a}}(f)= & \sum_{d=1}^{D} P_{V_{a_{d}} V_{a_{d}}}(f) \\
= & \sum_{d=1}^{D}\left|H_{d}\left(Z_{\ell}, f\right)\right|^{2} \\
& \times\left|\frac{Z_{i_{d}}\left(Z_{\ell}, f\right)}{Z_{i_{d}}\left(Z_{\ell}, f\right)+Z_{d}(f)}\right|^{2} P_{V_{s_{d}} V_{s_{d}}}(f)\left[\mathrm{V}^{2} / \mathrm{Hz}\right],
\end{aligned}
$$

where $Z_{i_{d}}\left(Z_{\ell}, f\right)$ is the input impedance of the power delivery network at the port where the $d$ th appliance is connected. All other quantities in (5) are consistent with the notation of Figure 1. Furthermore, we note that we account for the thermal noise contribution due to the real part of $Z_{d}(f)$ in the resistive noise term. Toward the receiver, the active noise injected by the $d$ th device is attenuated by $H_{d}\left(Z_{\ell}, f\right)$ and, further, by an attenuation factor that is a function of the input impedance $Z_{i_{d}}\left(Z_{\ell}, f\right)$. Both $H_{d}\left(Z_{\ell}, f\right)$ and $Z_{i_{d}}\left(Z_{\ell}, f\right)$ depend on the load impedance $Z_{\ell}(f)$. It follows that the design of the load impedance has an impact on the received active noise.

Now, we introduce the following approximations. We assume that $P_{V_{s_{d}} V_{s_{d}}}(f)$ is identical for all appliances, that is, $P_{V_{s_{d}} V_{s_{d}}}(f)=P_{V_{w} V_{w}}(f)$ for all $d \in\{1, \ldots, D\}$, and we neglect the source impedance, that is, we let $Z_{d}(f)=0$. In this respect, we point out that by letting $Z_{d}=0$, we follow a 
conservative approach because, in such a case, we experience the highest level of noise at the receiver.

In Section 5, we show that the optimal receiver impedance is independent from $P_{V_{w} V_{w}}(f)$. However, the value of $P_{V_{w} V_{w}}(f)$ is fundamental to obtain realistic values of SNR. To determine $P_{V_{w} V_{w}}(f)$, we explicit the quantity as follows:

$$
P_{V_{w} V_{w}}(f)=\frac{P_{V_{n} V_{n}}(f)-P_{V_{r} V_{r}}(f)-P_{V_{\ell} V_{\ell}}(f)}{\sum_{d=1}^{D}\left|H_{d}\left(Z_{\ell}, f\right)\right|^{2}}\left[\mathrm{~V}^{2} / \mathrm{Hz}\right],
$$

and we exploit the experimental results in [12]. Basically, $P_{V_{n} V_{n}}$ is the total additive background noise experienced at the receiver port. In [12], the total additive background noise experienced at the receiver port was measured and modeled in power terms for the reference load of $50 \Omega$. The model reads

$$
P_{\mathrm{bck}}(f)=\frac{1}{f^{2}}+10^{-15.5}[\mathrm{~mW} / \mathrm{Hz}]
$$

To be consistent with the measurement setup in [12], in (6), we let $Z_{\ell}$ be constant and equal to $50 \Omega$ and we substitute $P_{V_{n} V_{n}}$ with

$$
P_{V_{n} V_{n}}(f)=0.05 \cdot P_{\mathrm{bck}}(f)\left[\mathrm{V}^{2} / \mathrm{Hz}\right] \text {, }
$$

that is, the $\mathrm{V}^{2} / \mathrm{Hz}$-representation of the measured noise PSD in (7), where the coefficient 0.05 allows for the conversion from $\mathrm{dBm} / \mathrm{Hz}$ to $\mathrm{V}^{2} / \mathrm{Hz}$. Furthermore, in (6), we compute $P_{V_{r} V_{r}}(f)$ and $P_{V_{\ell} V_{\ell}}(f)$ as described in Sections 4.2 and 4.3, respectively.

4.2. Resistive Noise. Resistive elements introduce thermal noise [13]. The noisy impedance $Z(f)$ can be modeled as a thermal noise voltage source and a noiseless impedance. The PSD of the amplitude of the thermal noise reads

$$
P_{V_{Z} V_{Z}}(f)=4 k T \mathscr{R}\{Z(f)\}\left[V^{2} / H z\right],
$$

where $k=1.38 \cdot 10^{-23} \mathrm{~J} / \mathrm{K}$ is the Boltzmann constant, $T=$ $290 \mathrm{~K}$ is the absolute temperature, and $\mathscr{R}\{\cdot\}$ denotes the real operator. The branches and the nodes of the power delivery network are not ideal and they show a resistive component that contributes to the thermal noise at the receiver. Further sources of thermal noise are the internal impedance of both the transmitter and the household appliances. Now, we point out that the power delivery network between the transmitter and the receiver port can be modeled as a passive two-port network. We can gather the thermal noise contributions into the resistive noise PSD that can be obtained from the network impedance at the receiver port $Z_{o}(f)$. In this respect, we point out that an alternative description of the noise generated by the network can be obtained in terms of noise figure. For further details, we refer to [14]. In Figure 3(b), we show the equivalent network model from which we obtain

$$
P_{V_{r} V_{r}}(f)=\left|\frac{Z_{\ell}(f)}{Z_{\ell}(f)+Z_{o}(f)}\right|^{2} 4 k T \mathscr{R}\left\{Z_{o}(f)\right\}\left[\mathrm{V}^{2} / \mathrm{Hz}\right]
$$

and we remark that $Z_{o}(f)$ is a function of the internal impedance of the transmitter and the loads.

4.3. Receiver Noise. We isolate the thermal noise due to the receiver from the contribution due to the rest of the network and we refer to it as receiver noise, namely, $V_{\ell}(f)$. The receiver noise is due to the parallel impedance that we propose to use as IMN before the first amplification stage of the frontend, that is, the VGA. The parallel impedance provides an SNR improvement as shown in Section 6, but it can be, in general, resistive and thus it may increase the thermal noise contribution.

We do not account for the noise contribution due to the VGA because the input impedance of the VGA is modeled as noiseless. In fact, all noise contributions due to the amplification stage are described by the noise figure of the AFE [15].

In Figure 3(c), we show the equivalent model of the noisy receiver impedance. We model the PSD of $V_{\ell}(f)$ as

$$
P_{V_{\ell} V_{\ell}}(f)=\left|\frac{Z_{o}(f)}{Z_{o}(f)+Z_{\ell}(f)}\right|^{2} 4 k T \cdot \mathscr{R}\left\{Z_{\ell}(f)\right\}\left[V^{2} / H z\right] .
$$

From (11), we note that the PSD of the amplitude of the receiver noise is lower than the actual noise generated by the impedance $Z_{\ell}(f)$ due to the presence of the voltage divider term.

4.4. Final Model. We now explicit the dependencies of the SNR from the impedance of the receiver. To this aim, we proceed as follows. Firstly, we formulate the CFR and the output impedance of the power delivery network as a function of the chain-matrix parameters, that is, [16]

$$
\begin{gathered}
H_{i}\left(Z_{\ell}, f\right)=\left(A_{i}(f)+\frac{B_{i}(f)}{Z_{\ell}(f)}\right)^{-1}, \\
Z_{o}(f)=\frac{B_{u}(f)+D_{u}(f) Z_{s}(f)}{A_{u}(f)+C_{u}(f) Z_{s}(f)}[\Omega],
\end{gathered}
$$




$$
\Gamma\left(Z_{\ell}\right)=\frac{\left|\left(A_{u}+\frac{B_{u}}{Z_{\ell}}\right)^{-1}\right|^{2} P_{V_{t} V_{t}}}{\sum_{d=1}^{D}\left|\left(A_{d}+\frac{B_{d}}{Z_{\ell}}\right)^{-1}\right|^{2} P_{V_{w} V_{w}}}+\underbrace{\left|\frac{Z_{\ell}}{\frac{B_{u}+D_{u} Z_{s}}{A_{u}+C_{u} Z_{s}}+Z_{\ell}}\right|_{\text {resistive noise }}^{\mid 2} 4 k \mathscr{R}\left\{\frac{B_{u}+D_{u} Z_{s}}{A_{u}+C_{u} Z_{s}}\right\}}_{\text {active noise }}+\underbrace{\left|\frac{\frac{B_{u}+D_{u} Z_{s}}{A_{u}+C_{u} Z_{s}}}{\left.\frac{B_{u}+D_{u} Z_{s}}{A_{u}+C_{u} Z_{s}}\right|^{2}}\right|^{2} 4 k \mathscr{R}\left\{Z_{\ell}\right\}}_{\text {receiver noise }},
$$

where $i=u, d$, and $A_{i}, B_{i}, C_{i}$ and $D_{i}$ are the parameters of the transmission matrix that describes the link $i$. Then, we substitute (3), (4), and (12) in (1), and we replace the PSD of the noise terms with the correspondent expressions (5), (10) and (11), where we explicit $Z_{o}(f)$ according to (13). In (14), we report the resultant SNR expression. We neglect the frequency dependency for notation simplicity.

\section{SNR Optimization Problem}

We formulate the SNR optimization problem as follows:

$$
\begin{gathered}
Z_{\text {opt }}(f)=\underset{Z_{\ell}(f)}{\operatorname{argmax}}\left\{\Gamma\left(Z_{\ell}, f\right)\right\} \\
\text { s.t. } \quad x(f) \geq 0, \\
y(f) \in \mathbb{R},
\end{gathered}
$$

where $\mathbb{R}$ denotes the set of real numbers, and we introduce the notation $x(f)=\mathscr{R}\left\{Z_{\ell}(f)\right\}$ and $y(f)=\mathscr{I}\left\{Z_{\ell}(f)\right\}$, with $\mathscr{I}\{\cdot\}$ being the imaginary operator. We also highlight that the SNR is dependent on $Z_{\ell}$ and $f$. We start from (14) and, according to the derivations that we report in the Appendix, we obtain

$$
\Gamma(x, y)=\frac{P_{V_{t} V_{t}}}{P_{a}(x, y)+P_{r}(x, y)},
$$

where

$$
\begin{gathered}
P_{a}(x, y)=\sum_{d=1}^{D} \frac{f_{u}(x, y)}{f_{d}(x, y)} P_{V_{w} V_{w}}, \\
P_{r}(x, y)=4 k T\left(\zeta_{u}+\frac{\left|B_{u}\right|^{2}}{x^{2}+y^{2}} x\right), \\
f_{i}(x, y)=\left|A_{i}\right|^{2}\left(x^{2}+y^{2}\right)+2 \zeta_{i} x+2 \varsigma_{i} y+\left|B_{i}\right|^{2}, \\
\zeta_{i}=\mathscr{R}\left\{B_{i} A_{i}^{*}\right\}, \\
\varsigma_{i}=\mathscr{I}\left\{B_{i} A_{i}^{*}\right\},
\end{gathered}
$$

and $i=u, d$. Furthermore, we remark that all quantities in (16)-(21) are a function of the frequency and we neglect the dependence only for notation simplicity. Experimentally, we have observed that $\zeta_{i} \geq 0$, while $\zeta_{i}$ can be either positive or negative.

Now, we focus on the denominator in (16) and we note the following. Firstly, the active noise is described by the first term, while the contributions of the resistive and receiver noise are gathered in the second term. Secondly, the receiver noise contribution in $P_{r}(x, y)$ does not increase monotonically as a function of $x$. Furthermore, $P_{r}(x, y)$ can be neglected because it is always significantly lower than $P_{a}(x, y)$ for realistic noise scenarios. In fact, $P_{r}(x, y)$ is dominated by the Boltzmann constant $k$, and it assumes values in the order of $10^{-23}$. Indeed, $P_{V_{a} V_{a}}$ assumes values in the order of $10^{-17}$, according to the derivations of Section 4.1. Therefore, we can approximate the SNR as

$$
\Gamma(x, y) \approx \frac{1}{\sum_{d=1}^{D} f_{u}(x, y) / f_{d}(x, y)} \cdot \frac{P_{V_{t} V_{t}}}{P_{V_{w} V_{w}}} .
$$

We substitute (22) into (15) and we study the concavity of the maximization problem. In general, the expression in (22) is not concave in the domain of $x$ and $y$ that we consider. In detail, the functions $f_{i}(x, y)$ are convex. The ratio of convex functions is, in general, not convex. Similarly, the sum of nonconvex functions is, in general, not convex. Therefore, the denominator term in (22) is, in general, not convex. It follows that the maximization problem is not concave [17].

Consequently, we performed the exhaustive search to identify the optimal pair $(x, y)$ for each frequency bin, as described in Section 6. From simulation results, we obtained that the optimal receiver resistance is identically null in the entire frequency range, that is, $x=0$. Therefore, by letting $x=0$ in (15), we note that the domain of the maximization problem can be limited to the set of values $y=\{\hat{y}\}$ for which

$$
\frac{\partial \Gamma(x=0, y)}{\partial y}=0
$$

\section{Numerical Results}

We have studied the optimal receiver impedance in real networks. To this aim, we carried out a measurement campaign in Italy and we collected more than 1200 channel responses. We considered three sites, with 11, 23, and 26 outlets, respectively. We followed an exhaustive approach. For each site, we performed measurements between all pairs of available outlets, where no loads were connected.

We performed measurements in the frequency domain. We deployed a vector network analyzer (VNA) in combination with broadband couplers and extension cables. We removed the effect of the couplers and the cables from the measures to obtain the actual scattering parameters of the 
PLC channel. We connected the VNA to the network through coaxial cables and broadband couplers. Couplers protect the equipment from the mains and they show an attenuation of $50 \mathrm{~dB}$ at the mains frequency, and lower than $5 \mathrm{~dB}$ up to $100 \mathrm{MHz}$.

We calibrated the VNA when only the cables were connected and we removed the effect of the couplers by exploiting the chain rule of the ABCD matrices. To this aim, we characterized the couplers in terms of $A B C D$ matrices. The procedure proved to be the most reliable.

From the measured scattering parameters, we computed the CFR. In Figure 4, we provide the results. In different subplots, we show the channels from the three sites. Furthermore, we report the average profile (dashed line). In all cases the CFR exhibits a frequency decreasing behavior. In sites 2 and 3 , that is, the largest premises, the average attenuation is greater than in site 1, that is, a small urban flat. For the statistical characterization of the channels, we refer to [18], where we describe the channels in terms of the main metrics, and we study the probability density function of the CFR as a function of frequency. Furthermore, we point out that herein we consider a restricted set of the measurements in [18].

From the measured scattering parameters, we also computed the output impedance. We define it as in (13), and we note that it can be interpreted as the network impedance at the receiver port when the impedance $Z_{s}$ is connected to the transmitter port [18]. Hence, $Z_{o}$ is a function of the source impedance $Z_{s}$. In our case, $Z_{s}=0 \Omega$. We denote the real and imaginary component of $Z_{o}(f)$ with $R_{o}(f)$ and $X_{o}(f)$, respectively. To analyze the results, in Figure 5, we report the statistics in quantile terms. We focus on three probability values, that is, $\alpha=10,50$, and $90 \%$, and we plot the quantiles $q_{\Lambda, \alpha}(f)$ that corresponds to $\operatorname{Pr}\left[\Lambda(f) \leq q_{\Lambda, \alpha}(f)\right]=\alpha$, where $\Lambda \in\left\{R_{o}, X_{o}\right\}$ and $\operatorname{Pr}[\cdot]$ denotes the probability. Interestingly, we can note that the resistive component is more spread in the lower frequency range, while the reactive component exhibits statistically an inductive-like behavior; that is, it increases with frequency.

The measured values of $H_{d}, H_{u}$, and $Z_{o}$ have been substituted in (14). By exploiting measurements, we obtain realistic results, although we introduced the following approximations. We neglected the impact of the internal impedance of the noise sources on $H_{u}$ and $H_{d}$, and the impact of the internal impedance of the transmitter on $H_{d}$. In fact, when we performed measurements, no loads were connected to the available outlets. The household appliances were connected to the remaining outlets. Hence, according to the model of Figure 1, the measures resemble the case of no loads connected to the transmitter and the $D$ noise ports. Similar considerations hold true for the measurements of the output impedance.

For the analysis of the effect of the optimal impedance, we focus on the 1.5-100 MHz frequency range and the resolution of the measurements is $\Delta=500 \mathrm{kHz}$. In the following, we adopt the discrete frequency representation, and we denote with $l$ the frequency sample $f=l \Delta$. Furthermore, $f_{1}=$ $N_{1} \Delta=1.5 \mathrm{MHz}$ and $f_{2}=N_{2} \Delta=100 \mathrm{MHz}$.

We carried out an exhaustive search of the optimal receiver impedance for the measured channels. We limited

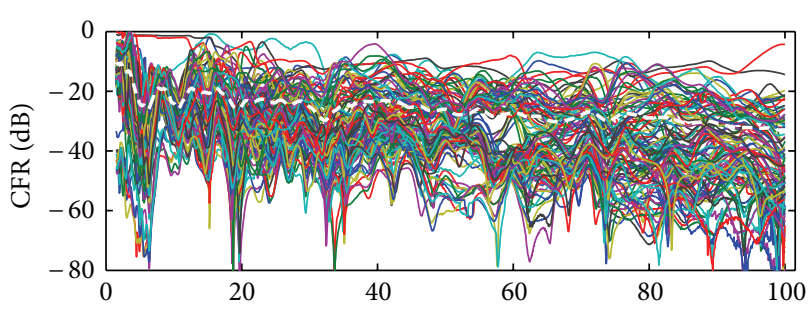

(a) Frequency $(\mathrm{MHz})$

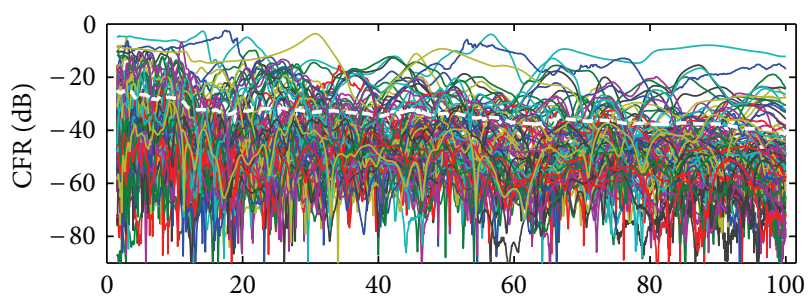

(b) Frequency $(\mathrm{MHz})$

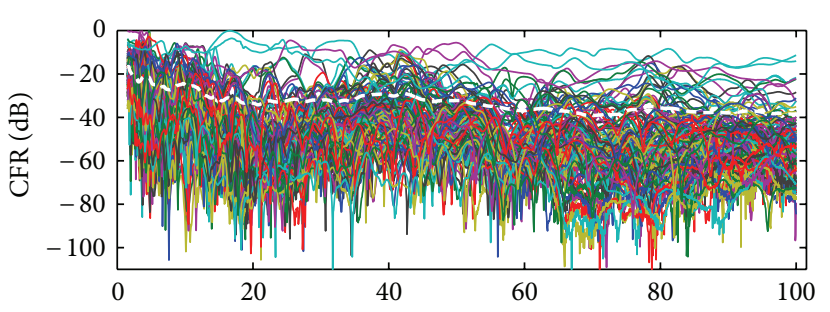

(c) Frequency $(\mathrm{MHz})$

FIGURE 4: Measured channel frequency responses. From top to bottom, sites 1, 2, and 3. In all cases, the mean profile is also shown (dashed line).

the search domain to $\{0,1 \mathrm{k} \Omega\}$ and to $\{-1 \mathrm{k} \Omega, 1 \mathrm{k} \Omega\}$ for the resistive and the reactive component, respectively. In both cases, the resolution is $1 \Omega$. In Figures 6 and 7, we show the quantiles of the components of $Z_{\text {opt }}(f)$ as a function of the frequency. We limit the plot to three probability values, that is, $\alpha=20,50$, and $80 \%$. Figure 6 shows that the resistive component of the optimal receiver impedance is equal to 0 for all realizations and frequencies. We speculate that this result is determined by the nonnegative behavior of the coefficients $\zeta_{i}$ in (18)-(20). Indeed, Figure 7 shows that the reactive component of the optimal receiver impedance exhibits a strong frequency-dependent behavior. Furthermore, the median value of the reactive component is close to 0 . It follows that the optimal impedance does not exhibit a prominent inductive-like or capacitive-like behavior.

In Figures 6 and 7, we compare the statistics of the optimal receiver impedance to the statistics of the matched impedance, that is, the impedance that is designed to fulfil the power matching conditions. To this aim, we show the quantiles of the receiver impedance under the power matching assumption, namely, $Z_{\mathrm{pmi}}=Z_{o}^{*}$. We focus on the probability values $\alpha=20,50$ and $80 \%$. Differently from the optimal case, the resistive component of the matched impedance is nonzero and, by definition, it is equal to the 


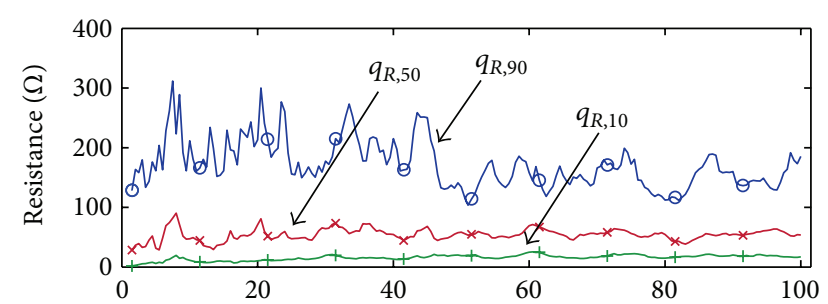

(a) Frequency $(\mathrm{MHz})$

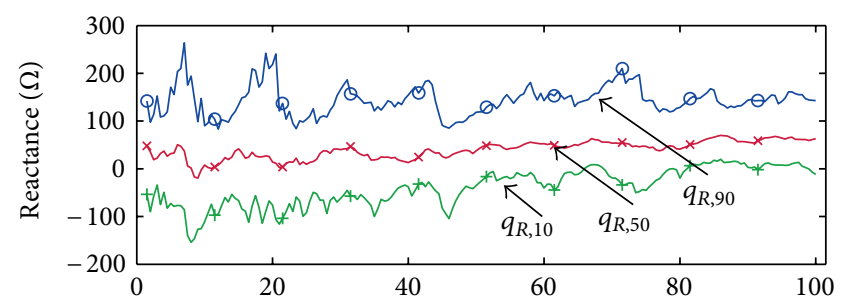

(b) Frequency $(\mathrm{MHz})$

FIGURE 5: Quantiles of the resistive (a) and reactive (b) component of the output impedance. Three probability values are considered, that is, 10,50 and $90 \%$.

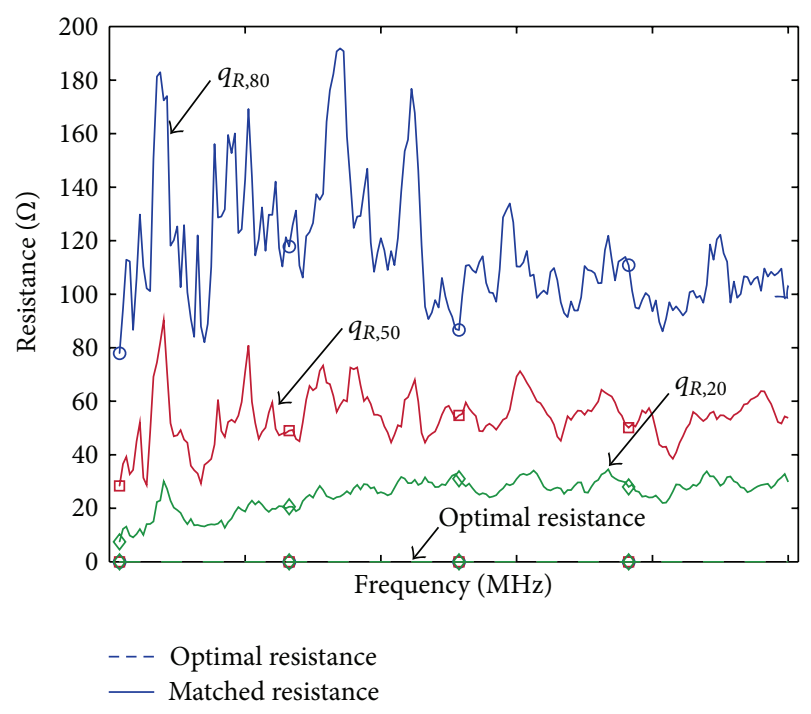

FIGURE 6: Quantiles of the resistive component of the optimal and power matched receiver impedance. Three probability values are considered, that is, 20,50 , and $80 \%$.

resistive component of the output impedance. Indeed, the statistics of the reactive component of $Z_{\mathrm{pmi}}$ and $Z_{\mathrm{opt}}$ are similar, but, for the matched impedance, the high-probability area is better confined around the median and the profiles of the quantiles associated to probabilities $\alpha=20$ and 80 are smoother in frequency. Finally, the median of the reactive component of the matched impedance overlaps the median of the reactive component of the optimal impedance.

Now, we study the SNR, namely, $\Gamma\left(Z_{\ell}, l\right)$. We compute it according to (14). We let the transmitted power (in dB terms) be

$$
P_{V_{t} V_{t}}(l)= \begin{cases}-63 \mathrm{dBV} / \mathrm{Hz} & \text { for } l \Delta \leq 30 \mathrm{MHz} \\ -93 \mathrm{dBV} / \mathrm{Hz} & \text { otherwise }\end{cases}
$$

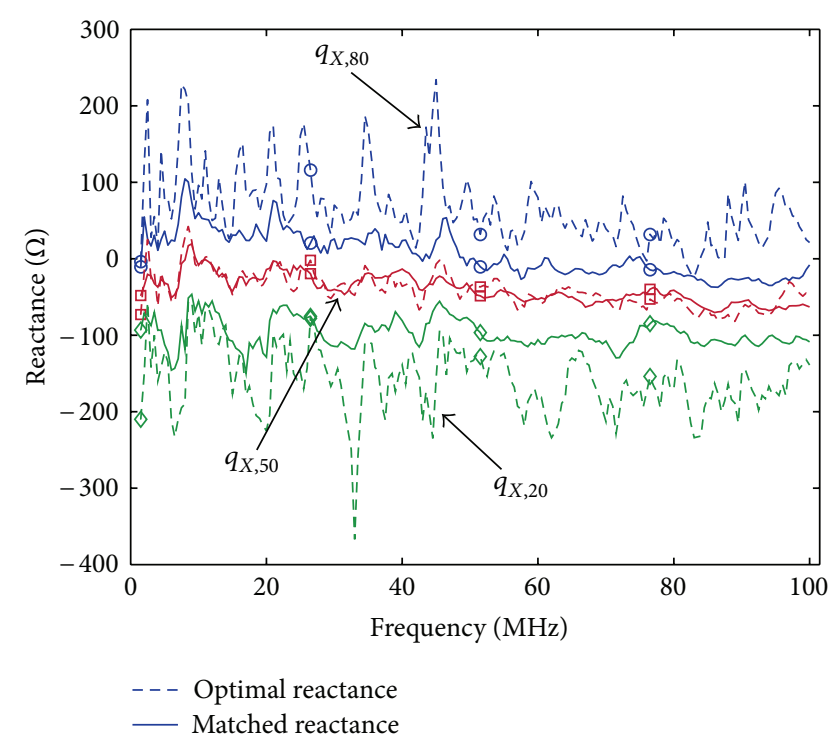

FIGURE 7: Quantiles of the reactive component of the optimal and power matched receiver impedance. Three probability values are considered, that is, 20,50 , and $80 \%$.

that is, the values that yield to a transmitted power of $-50 \mathrm{dBm} / \mathrm{Hz}$ below $30 \mathrm{MHz}$, and $-80 \mathrm{dBm} / \mathrm{Hz}$ beyond $30 \mathrm{MHz}$ on a reference load of $50 \Omega$. Basically, we shape $P_{V_{t} V_{t}}$ as described in [12], and not as directly specified by the EMC norms because the latter do not target the frequency range beyond $30 \mathrm{MHz}$ yet. Furthermore, we determine $P_{V_{w} V_{w}}$ as described in Section 4.1.

We compute the SNR when the receiver impedance is optimal, matched in power, or constant in frequency and equal to $Z_{50}=50 \Omega$. We assume the latter to be the reference case, and we obtain the SNR improvements provided by the use of $Z_{\mathrm{opt}}$ and $Z_{\mathrm{pmi}}$ with respect to $Z_{50}$, that is, $\Delta \Gamma\left(Z_{\kappa}, l\right)=\left(\Gamma\left(Z_{\kappa}, l\right)\right)_{\mathrm{dB}}-\left(\Gamma\left(Z_{50}, l\right)\right)_{\mathrm{dB}}$ where $\kappa \in\{$ opt, pmi $\}$. Note that $\Delta \Gamma$ is formulated as the difference of the SNR in $\mathrm{dB}$ terms. Hence, we study the statistics of $\Delta \Gamma\left(Z_{\kappa}, l\right)$. In Figure 8, we show the fitted profiles of the quantiles of $\Delta \Gamma\left(Z_{\kappa}, l\right)$ associated to the probability values 20,50 , and $80 \%$. Impedance adaptation techniques are more beneficial in the lower frequency range, where the optimal impedance and the power matched impedance provide, in half of the cases, up to 4 and $1 \mathrm{~dB}$ of improvement, respectively. In the higher frequency range and for half of the cases, the matched impedance does not provide any improvement, while the optimal impedance ensures an SNR gain of approximately $1 \mathrm{~dB}$. Finally, we note that, according to the formulation in (14), the matched impedance can even reduce the SNR with respect to the case of using $Z_{50}$ (see $\operatorname{Pr}\left[\Delta \Gamma\left(Z_{\mathrm{pmi}}, l\right) \leq \gamma\right]=\alpha$ for $\alpha=20 \%$ in Figure 8).

Now, we study the performance in terms of achievable rate. We assume the transmitted signal and the noise to be Gaussian, and we define the achievable rate as follows:

$$
C(v)=\Delta \sum_{l=N_{1}}^{N_{2}} \log _{2}\left(1+\Gamma\left(Z_{v}, l\right)\right)[\mathrm{bps}]
$$




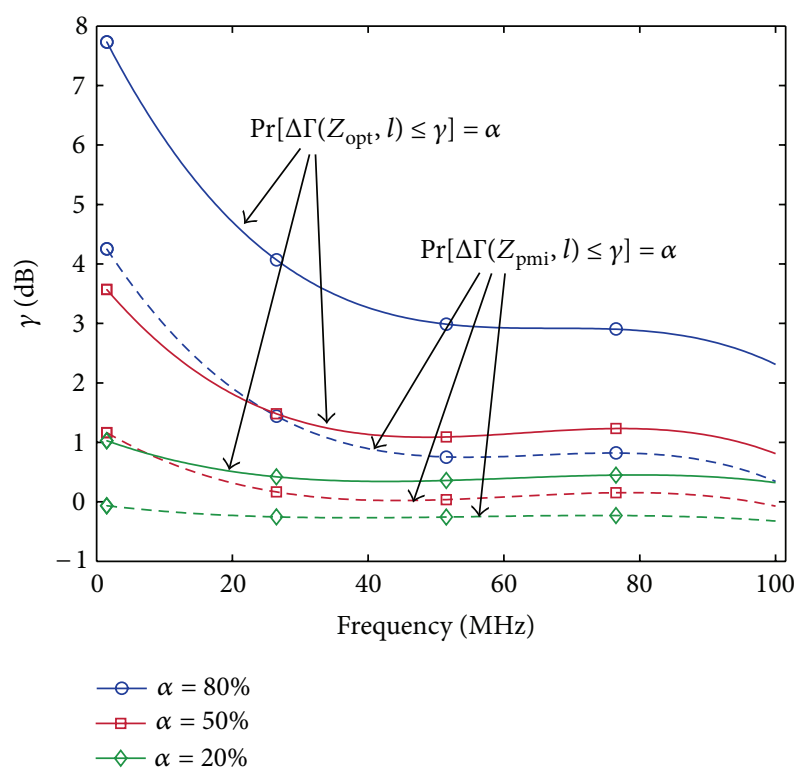

FIGURE 8: Best cubit fit of the SNR quantile profiles associated to the probability values $\alpha=20,50$, and $80 \%$.

where $v \in\{$ opt, pmi, 50\}, and we limit the transmitted PSD according to (24). In Figure 9(a), we show the complementary cumulative distribution function (C-CDF) of the achievable rate for the three receiver impedances that we consider. We magnify the plot for the probability values greater than 0.6. As expected, the optimal impedance outperforms the power matched impedance. As an example, $C$ (opt) exceeds $800 \mathrm{Mbps}$ in $83 \%$ of the cases, while $C$ (pmi) achieves the same rates in $77 \%$ of the cases. In a dual manner, with probability $0.9, C$ (opt) > $745 \mathrm{Mbps}$ while $C$ (pmi) > $695 \mathrm{Mbps}$, with an improvement of 7\%.

Now we quantify the improvement with respect to $Z_{50}$. We compute the achievable rate improvement as follows:

$$
\Delta C(\kappa)=100 \frac{C(\kappa)-C(50)}{C(50)}[\%],
$$

where $\kappa \in\{$ opt, pmi\}. In Figure 9(b), we show the cumulative distribution function (CDF) of $\Delta C(\kappa)$. The use of the optimal impedance turns into achievable rate improvements of up to $24 \%$. Interestingly, the power matching approach does not provide significant benefit with respect to $Z_{50}$. In this case, the improvement is lower than $5 \%$ in $90 \%$ of the cases. Furthermore, the matched impedance can even reduce the achievable rate. The reason is that the matched impedance maximizes the power of the received signal, but not necessarily the SNR.

6.1. High Receiver Impedance Design. In the previous section, we have shown the performance when the receiver impedance is optimal, matched in power or constant and equal to $50 \Omega$. It is also interesting to address the performance when the receiver impedance is high. In such case, we denote the receiver impedance with $Z_{\text {high }}$ and we let it be purely real and equal to $1 \mathrm{k} \Omega$, that is, the largest value that we admitted for the exhaustive search of the optimal impedance. We limit

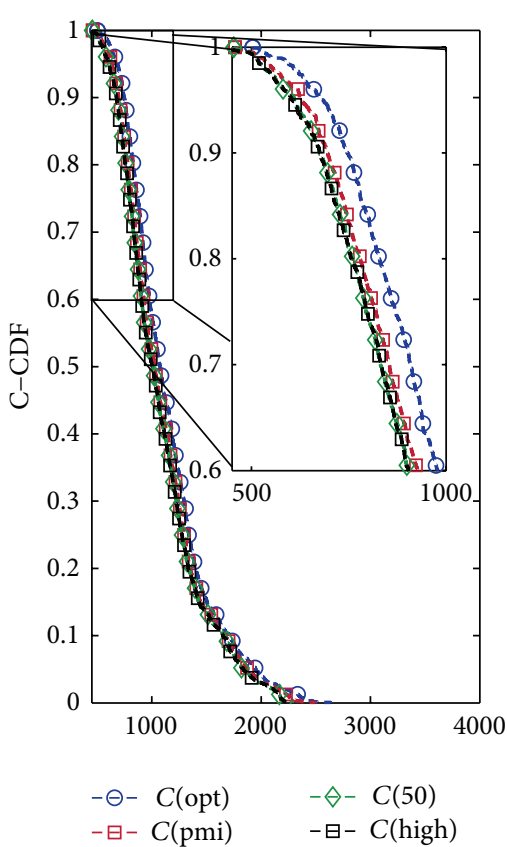

(a) Achievable Rate (Mbps)

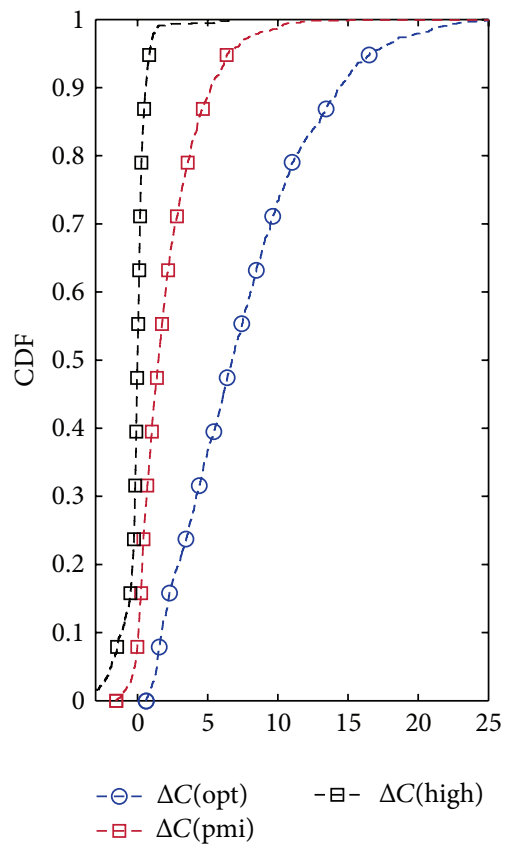

(b) Improvement (\%)

FIGURE 9: (a) C-CDF of the achievable rate when the receiver impedance is optimal, matched in power or constant and equal to $50 \Omega$ or $1 \mathrm{k} \Omega$. (b) $\mathrm{CDF}$ of the achievable rate improvement with respect to the case of $Z_{50}$.

the study to the achievable rate. We compute it as in (25), and we denote it with $C$ (high). Similarly, we compute the achievable rate improvement as in (26), and we denote it with $\Delta C$ (high). In Figure 9, we show both the C-CDF of $C$ (high) and the CDF of $\Delta C$ (high). As it can be noted, $Z_{\text {high }}$ does not provide any benefit with respect to $Z_{50}$ and the two solutions 
are equivalent in terms of achievable rate. Similar results can be obtained by letting $Z_{\text {high }}$ be large, and purely imaginary either positive or negative.

As a final remark, we note that the optimal impedance provides the best gain in terms of achievable rate. However, it is rather complex to be implemented in practice. Therefore, more simpler though suboptimal solutions, as $Z_{50}(f)$ and $Z_{\text {high }}(f)$, are often used.

\section{Conclusions}

We have investigated the optimal design of the receiver input impedance for the maximization of the SNR in broadband PLC. We have firstly presented a comprehensive description of the power delivery network. We have modelled the contribution of the household appliances on the noise at the receiver port. Then, we have discussed the front-end design for broadband PLC transceivers. We have pointed out that the amplitude (and not the power) of the received signal is important for data communication purposes.

Therefore, we have formulated the SNR in amplitude terms, and we have modelled the noise as the sum of four contributions, namely, the active, resistive, receiver, and coupled noise. Basically, the active noise is the noise that is injected by the household appliances, the resistive noise accounts for the thermal noise due to the resistive components of the network, the receiver noise is the thermal noise due to the receiver impedance, and the coupled noise is the noise that couples into the wirings, for example, due to broadcast radios.

We have studied the dependency of the SNR from the receiver impedance and we have highlighted the latter impacts not only on the amplitude of the useful signal, but also on the amplitude of the noise at the receiver port. Hence, we have found the optimal receiver impedance that maximizes the SNR. To this aim, we have exploited the results of an experimental measurement campaign where we have collected the scattering parameters of PLC channels in real home grids. From the measurement results, we have found that the optimal impedance is purely reactive and not equal to that obtained according to the power matching approach, namely, the matched impedance. We have compared the performance of the optimal impedance to that of the matched impedance in terms of SNR and achievable rate for reallife scenarios. We have assumed the performance of a $50-\Omega$ receiver as the reference case and we have found that while the matched impedance can even reduce the performance, the optimal impedance provides improvements up to $24 \%$ in terms of achievable rate. Finally, we have shown that when the receiver exhibits a high input impedance, the achievable rate is close to that obtained with a $50-\Omega$ receiver. Therefore, the two solutions are equivalent in performance.

\section{Appendix}

\section{SNR Derivations}

We aim to simplify the SNR formulation in (14) to obtain (16). We neglect the frequency dependency for notation simplicity, and we proceed as follows. Firstly, we introduce the notation $x=\mathscr{R}\left\{Z_{\ell}\right\}$ and $y=\mathscr{I}\left\{Z_{\ell}\right\}$. Then, we substitute $Z_{\ell}$ in (12) with $x+j y$, where $j$ is the imaginary unit, to obtain

$$
\left|H_{i}(f)\right|^{2}=\frac{x^{2}+y^{2}}{\left|A_{i}\right|^{2}\left(x^{2}+y^{2}\right)+\left|B_{i}\right|^{2}+2 x \zeta_{i}+2 y \zeta_{i}}
$$

where $i=u, d, \zeta_{i}$ and $\varsigma_{i}$ are defined as in (20)-(21), and we exploited the following relations:

$$
\begin{aligned}
& \zeta_{i}=\mathscr{R}\left\{A_{i}\right\} \mathscr{R}\left\{B_{i}\right\}+\mathscr{I}\left\{A_{i}\right\} \mathscr{I}\left\{B_{i}\right\}, \\
& \varsigma_{i}=\mathscr{R}\left\{A_{i}\right\} \mathscr{I}\left\{B_{i}\right\}-\mathscr{I}\left\{A_{i}\right\} \mathscr{R}\left\{B_{i}\right\} .
\end{aligned}
$$

Now, we focus on the output impedance $Z_{o}$. When $Z_{s}=$ 0 , the real part of $Z_{o}$ reads

$$
\mathscr{R}\left\{Z_{o}\left(Z_{s}=0 \Omega\right)\right\}=\mathscr{R}\left\{\frac{B_{u}}{A_{u}}\right\}=\frac{\mathscr{R}\left\{B_{u} A_{u}^{*}\right\}}{\left|A_{u}\right|^{2}} .
$$

We exploit (A.4), and we explicit the resistive and the receiver noise in (14) as

$$
\begin{aligned}
P_{V_{r} V_{r}} & =\left|\frac{x+j y}{B_{u} / A_{u}+x+j y}\right|^{2} \\
& =\frac{\left|A_{u}\right|^{2}\left(x^{2}+y^{2}\right)}{\left|A_{u}\right|^{2}\left(x^{2}+y^{2}\right)+\left|B_{u}\right|^{2}+2 x \zeta_{u}+2 y \varsigma_{u}} \\
P_{V_{e} V_{\ell}} & =\left|\frac{B_{u} / A_{u}}{B_{u} / A_{u}+x+j y}\right|^{2} \\
& =\frac{\left|B_{u}\right|^{2}}{\left|A_{u}\right|^{2}\left(x^{2}+y^{2}\right)+\left|B_{u}\right|^{2}+2 x \zeta_{u}+2 y \varsigma_{u}},
\end{aligned}
$$

respectively. Finally, we substitute (A.1) and (A.5)-(A.6) in (14); we divide the numerator and the denominator by (A.1) and we obtain (16).

\section{References}

[1] F. J. Cañete, J. A. Cortés, L. Díez, and J. T. Entrambasaguas, "Analysis of the cyclic short-term variation of indoor power line channels," IEEE Journal on Selected Areas in Communications, vol. 24, no. 7, pp. 1327-1338, 2006.

[2] H. C. Ferreira, L. Lampe, J. Newbury, and T. G. Swart, Power Line Communications: Theory and Applications for Narrowband and Broadband Communications over Power Lines, Wiley \& Sons, New York, NY, USA, 2010.

[3] N. Taherinejad, R. Rosales, S. Mirabbasi, and L. Lampe, "On the design of impedance matching circuits for vehicular power line communication systems," in Proceedings of the IEEE International Symposium on Power Line Communications and Its Applications (ISPLC '12 ), pp. 322-327, March 2012.

[4] P. A. Janse van Rensburg and H. C. Ferreira, "Design of a bidirectional impedance-adapting transformer coupling circuit for low-voltage power-line communications," IEEE Transactions on Power Delivery, vol. 20, no. 1, pp. 64-70, 2005. 
[5] A. M. Tonello and F. Versolatto, "Bottom-up statistical PLC channel modeling-part I: random topology model and efficient transfer function computation," IEEE Transactions on Power Delivery, vol. 26, no. 2, pp. 891-898, 2011.

[6] Maxim, "Integrated powerline communication analog frontend transceiver and line driver," http://www.maximic.com/ datasheet/index.mvp/id/6333.

[7] P. G. M. Baltus and R. Dekker, "Optimizing RF front ends for low power," Proceedings of the IEEE, vol. 88, no. 10, pp. 15461559, 2000.

[8] J. Bauwelinck, E. de Backer, C. Mèlange et al., "A 1024-QAM analog front-end for broadband powerline communication up to $60 \mathrm{MHz}$," IEEE Journal of Solid-State Circuits, vol. 44, no. 5, pp. 1477-1485, 2009.

[9] B. Razavi, RF Microelectronics, Prentice Hall, 1998.

[10] C. Bowick, RF Circuit Design, Newnes, 1982.

[11] L. W. Couch, Digital and Analog Communication Systems, Prentice Hall, 1997.

[12] R. Hashmat, P. Pagani, and T. Chonavel, "MIMO communications for inhome PLC networks: measurements and results up to $100 \mathrm{MHz}$," in Proceedings of the IEEE International Symposium on Power Line Communications and Its Applications (ISPLC '10), pp. 120-124, March 2010.

[13] A. Papoulis, Probability, Random Variables, and Stochastic Processes, McGraw-Hill, 1991.

[14] H. T. Friis, "Noise figures of radio receivers," Proceedings of the IRE, vol. 32, no. 7, pp. 419-422, 1944.

[15] W. F. Egan, Practical RF System Design, John Wiley \& Sons, 2003.

[16] D. M. Pozar, Microwave Engineering, Wiley Academy, 2004.

[17] S. Boyd, Convex Optimization, Cambridge University Press, 2004.

[18] A. M. Tonello and F. Versolatto, "PLC channel characterization up to $300 \mathrm{MHz}$ : frequency response and access impedance," in Proceedings of the IEEE Global Communications Conference (GLOBECOM '12), December 2012. 

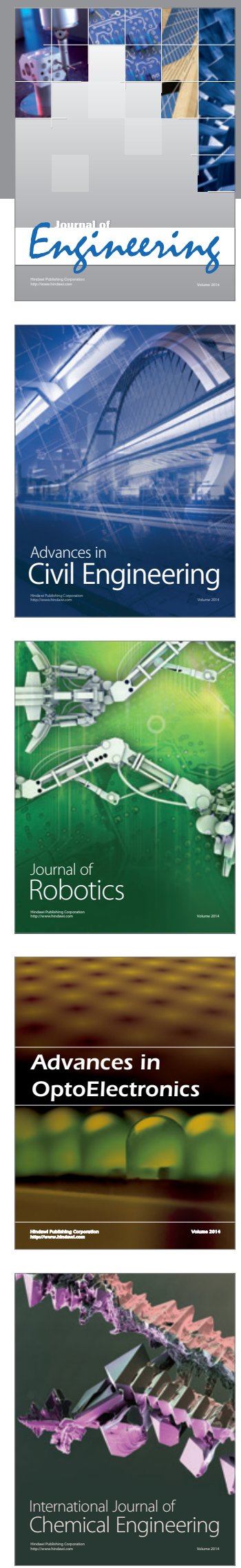

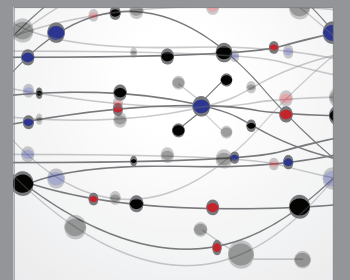

The Scientific World Journal
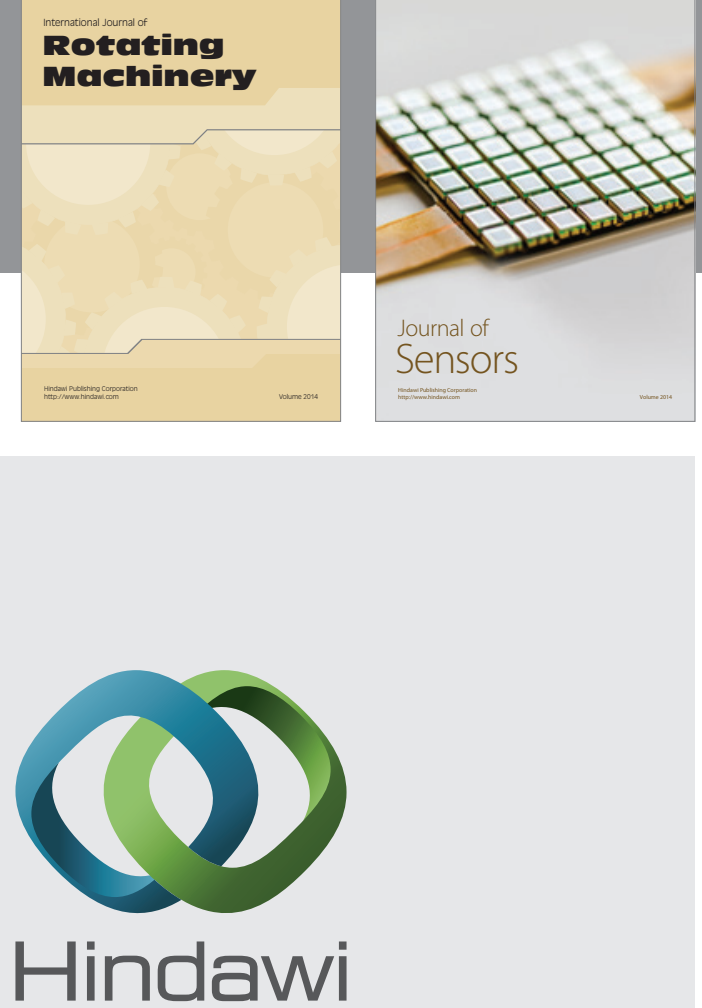

Submit your manuscripts at http://www.hindawi.com
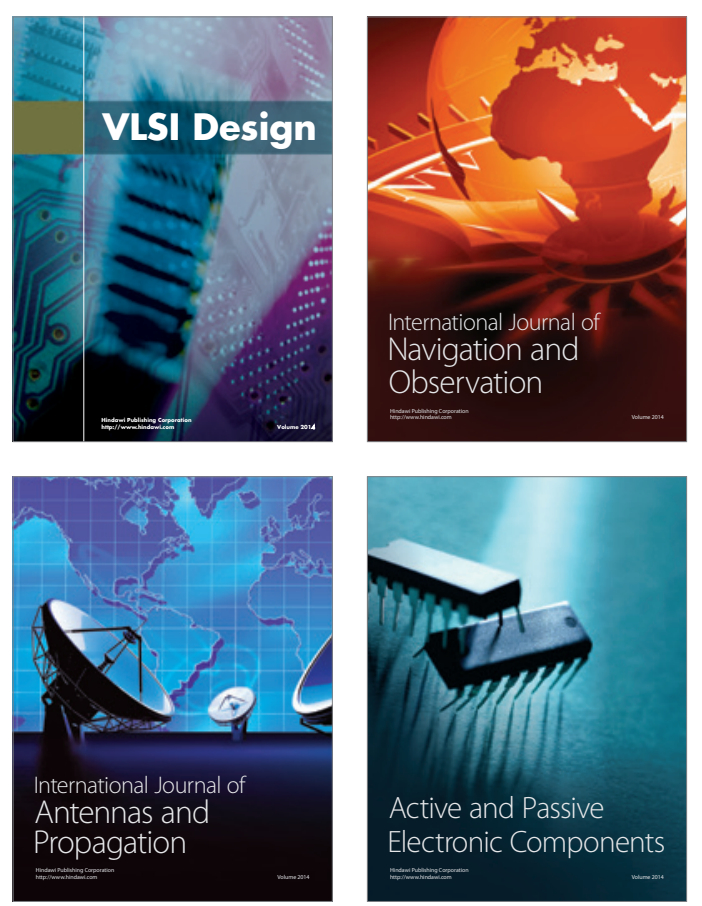
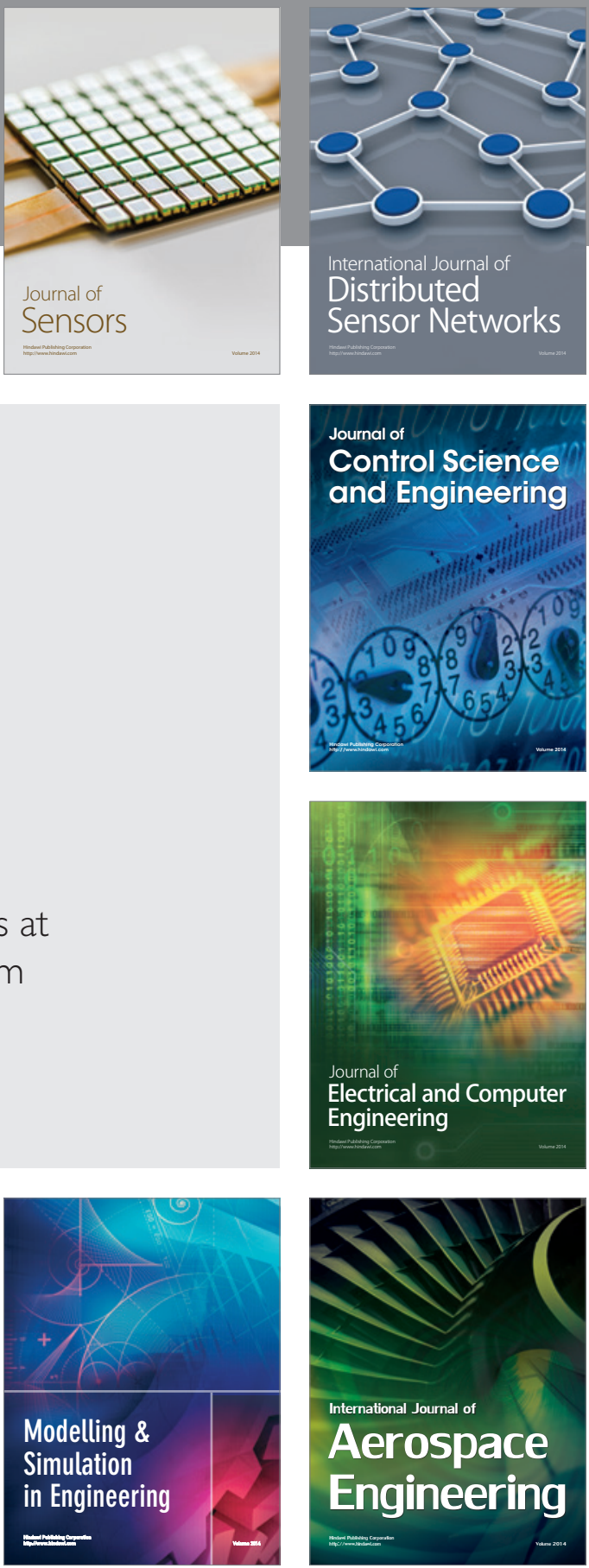

Journal of

Control Science

and Engineering
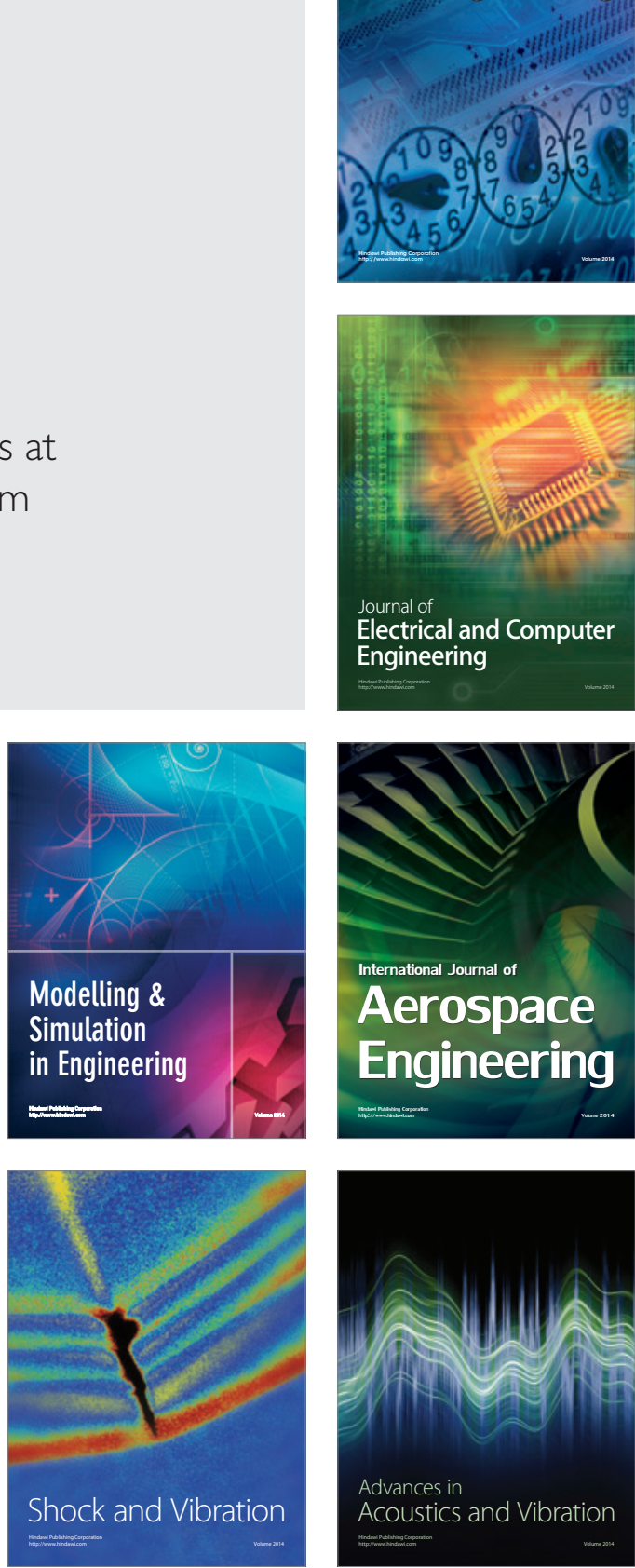